\title{
An Analysis on the Impact of Video Traffic over Wireless Visual Sensor Network under Mannasim Sensor Network Architecture
}

\author{
V. Kanakaris ${ }^{1}$, G.A. Papakostas ${ }^{2}$, D. Bandekas ${ }^{3}$ \\ ${ }^{1}$ MEASUREMENTS-Lab, Department of Electrical Engineering, \\ TEI of Eastern Macedonia \& Thrace, GR-65404 Agios Loukas, Kavala, Greece \\ ${ }^{2}$ HUMAIN-Lab, Department of Computer and Informatics Engineering, \\ TEI of Eastern Macedonia \& Thrace, GR-65404 Agios Loukas, Kavala, Greece \\ ${ }^{3}$ MEASUREMENTS-Lab, Department of Electrical Engineering, \\ TEI of Eastern Macedonia \& Thrace, GR-65404 Agios Loukas, Kavala, Greece \\ 1vkanak@teiemt.gr \\ 2gpapak@teikav.edu.gr \\ 3dbandek@teikav.edu.gr
}

\begin{abstract}
The monitoring capability of a Wireless Sensor Network (WSN) constitutes an important feature making its application multidisciplinary. In a common WSN, the network handles only simple scalar data and thus it is sufficient for complex applications such as object detection, surveillance, image recognition, localization, and tracking. Therefore, in addition to normal sensing network infrastructures, the functionality of a WSN can be improved by adding vision capabilities. The existing hardware and software specifications of a typical WSN node are determined only for handling simple tasks. The main objective of this work is to extend the WSN to a Visual Sensor Network (VSN) by adding some visual sensor nodes in the traditional WSN infrastructure and study the video traffic impact to the overall performance with respect to different metrics. For these purposes, a sensor network with some Mica2 Motes with added visual sensors acting as cluster heads as well as routers was simulated herein. The generated data by the Mica Motes and the visual sensors nodes will be collected by separate sink nodes. The performance of the network will be studied with respect to different number of simultaneous video connections. The impact of video traffic over the scalar data flow as well as the overall performance will be analyzed with suitable metrics.
\end{abstract}

Keywords - Visual Sensor; Video Traffic; Wireless Sensor; Network Performance

\section{INTRODUCTION}

\section{A. Wireless Sensor Networks (WSNs)}

One of the main function of a Wireless Sensor Network (WSN) is to monitor and distribute data/information “On-Demand” [1]. This specific feature can be used in a variety of applications related to industrial, military, health, areas after disaster, environmental monitoring. Most of the above mentioned applications provide information which is crucial and need to have reliability. Wireless Sensor Networks consisted of autonomous sensor nodes, which can monitor constantly the occurred phenomena. They can be deployed in situations where the resources are limited, such as areas after an earthquake or contamination and battlefields. Each sensor of a WSN can be scheduled to run special services [1],[2],[4]. The sensor nodes are operated systems using low processing power, allowing them to perform tasks and communicate with sink (central) or adjacent nodes [3].

B. Visual sensor networks (VSNs)

On the other hand, Visual Sensor Networks (VSNs) [5]-[7] consisted of low power visual sensors, each one equipped with an image sensor, CPU and wireless transceiver. Visual sensor networks can be used in many applications, such as security, and image identification. The basic difference between WSNs and VSNs is the dimension of the captured information about the environment. The former captures information as 1-D signal, while the latter as 2-D in the form of a digital image. Therefore, the data contain more information and need more process and analysis of VSNs compared to those provided by WSNs. The special features of VSNs are described in [6],[8] and we are going to present them in the following lines: Energy Consumption Demands Power usage in VSNs is higher compared to the conventional scalar wireless sensor networks.

Local Processing Procedure - By processing the image data locally/onboard the amount of the transmitted data is very low. Local processing may include simple as well as complex image and/or computer vision processing algorithms.

Real-Time Performance - On VSNs most applications demand a real-time communication between the camera nodes and sink, which means strict limits on the maximum allowable delay of data sources between the source node (camera) and the sink. 
Location and Orientation Information - Most of the algorithms regarding image processing algorithms need information about the position of the camera nodes and the orientation of the cameras.

Time Synchronization - A captured image may include meaningless information in cases that is not followed with the information regarding the time that the image was captured. That means that time synchronization is very essential on VSNs.

Data Storage - The cameras create large amounts of data over time, which need to be stored for later analysis.

Camera Interplay - Precise information regarding the position and orientation of the camera is vital for many algorithms used in VSNs. This information is achieved during the calibration phase by processing a set of special markers. The adjustment of cameras' settings can be performed in a processing center, which gathers all the special marks from each camera.

C. Performance of VSNs

The performance of a VSN depends on many factors which have already addressed from other researchers [6], [9]-[14]:

- expandability

- operating environment

- sensor deployment

- visual data transmission

- $\quad$ high bandwidth demand

- multimedia compression techniques

- image data processing

- error protection schemes

- fault tolerance

\section{SIMULATOR SELECTION}

There is a variety of simulators available for simulating wireless networks, but almost all the existing network simulators will not support WAN simulation completed. Moreover, on the authors' best knowledge there is not a commercial or freely available simulator for simulating all the features of VSNs. Most of the resent simulations based studies related with WSNs and VSNs, use NS-2 and OMNeT++. So, in this section we present and compare the NS-2 and OMNeT++.

A. Mannasim - Network Simulator (NS-2) [Simulator I]

The NS-2 is one of the most famous network simulators widely used in many researches. The NS-2 covers almost all of the IP protocols, but does not support all of the features needed to simulate a WSN [15],[16]. Specifically, the WSNs' characteristics that can be simulated by the NS-2 simulator are the following [15]-[17]:

- Wireless Channel

- Radio Models

- MAC Models

- Routing Models

- Energy Consumption Models

Mannasim extension [19] supports development and analysis of different WSNs applications. The main features that Mannasim supports, and will be used as initial approach in this research work in order to develop different sensor node types, are the following [19],[20]:

- Energy consumption for sensing and processing

- Number of instructions executed per seconds by its microcontroller

- Sensing interval regulation

- Data aggregation/dissemination methods

B. OMNeT++ [Simulator II]

Another notorious simulator is OMNeT++ [15]-[17], [19] which simulates wired and wireless network. The components that uses are based on $\mathrm{C}++^{+}$, although models use high-level language called NED1 (NEtwork Description language).

The OMNeT++ for WSNs simulation has an extension which called Castalia[17], [19]-[22] providing also a component to study the Intrusion Detection phenomenon. 
Table 1 [19], [22]-[25] presents in summary the specifications provided by Mannasim and Castalia frameworks.

Table 1. Features available in Mannasim and Castalia frameworks.

\begin{tabular}{|c|c|c|c|c|c|c|}
\hline Simulator & $\begin{array}{c}\text { Prog. } \\
\text { Languages }\end{array}$ & $\begin{array}{c}\text { Energy } \\
\text { consumption } \\
\text { model }\end{array}$ & MAC protocols & $\begin{array}{l}\text { Wireless } \\
\text { channel } \\
\text { models }\end{array}$ & $\begin{array}{c}\text { Visualizatio } \\
\text { n of } \\
\text { WSN/VSN } \\
\text { nodes }\end{array}$ & $\begin{array}{l}\text { GUI Based } \\
\text { Scenario } \\
\text { Generation } \\
\text { tool }\end{array}$ \\
\hline $\begin{array}{c}\text { Mannasim } \\
\text { under NS-2 }\end{array}$ & $\mathrm{C}++, \mathrm{OTcl}$ & $\begin{array}{l}\text { Extends linear } \\
\text { subtraction of } \\
\text { NS-2 }\end{array}$ & $\begin{array}{c}\text { IEEE } 802.11 \\
\text { MAC }\end{array}$ & $\begin{array}{l}\text { Free space } \\
\text { Two-ray } \\
\text { ground } \\
\text { reflection } \\
\text { Log normal } \\
\text { shadowing }\end{array}$ & $\begin{array}{l}\text { Simple } \\
\text { circular } \\
\text { Objects in } \\
\text { different } \\
\text { colours }\end{array}$ & $\begin{array}{c}\text { Provides a } \\
\text { Java based } \\
\text { GUI script } \\
\text { generator tool }\end{array}$ \\
\hline $\begin{array}{c}\text { OMNeT ++ } \\
\text { with } \\
\text { Castalia }\end{array}$ & $\mathrm{C}++, \mathrm{NED}$ & $\begin{array}{c}\text { Linear } \\
\text { subtraction }\end{array}$ & $\begin{array}{c}\text { Tunable MAC } \\
\text { T-MAC and S- } \\
\text { MAC IEEE } \\
\text { 802.15.4 MAC } \\
\text { Baseline BAN } \\
\text { MAC }\end{array}$ & $\begin{array}{c}\text { Free space } \\
\text { Log normal } \\
\text { shadowing } \\
\text { Temporal } \\
\text { variation } \\
\text { Additive } \\
\text { interference }\end{array}$ & $\begin{array}{c}\text { Different } \\
\text { Icons/Image } \\
\text { s for } \\
\text { different } \\
\text { kinds of } \\
\text { nodes }\end{array}$ & $\begin{array}{l}\text { Command line } \\
\text { based tool is } \\
\text { available for } \\
\text { random VSN } \\
\text { scenario } \\
\text { creation }\end{array}$ \\
\hline
\end{tabular}

We have test both of the above mentioned simulators. NS-2 is the most famous and powerful network simulation tool, since the number of researchers that use it has increased greatly over the last decade [29]. For example, 35 of the 80 simulation-based MANET papers published in the 2000-2005 ACM MobiHoc proceedings (i.e. about 43.8\%) used Ns-2 [29]. This happens because it is freely available, open source and includes detailed simulation capabilities of the major operations of such networks. The development efforts of the simulator have been supported by DARPA and NSF. Until recent years, only an insignificant percentage of researches published their papers based on OMNeT ++ .

We have decided to use the Mannasim extension of NS-2 for the proposed simulation study because of its popularity and the support that provides the user community. Moreover, we are only focusing on the transport and network layer issues of the VSN design, where NS-2 is well suited. In addition to that, the video traffic generator being used for the VSN simulation was originally written for NS-2. So, it is easily portable to Mannasim wireless sensor network simulations.

\section{MODELING THE VIDEO TRAFFIC AND VIDEO SENSOR NETWORK}

\section{A. The Simulated VSN Application Scenario}

We may assume the simulated network as a remote monitoring scenario such as a forest animal monitoring sensor network, a forest fire monitoring system or intrusion detection network used by military. Irrespective of the application, the main functionality of the network will be more or less the same. So here we explain the generic aspects of this network.

In the following WSN/VSN scenario, there are thirty-six normal sensor nodes (having capabilities equal to that of a Mica2 mote), which generate temperature data and periodically forward it to nine Cluster Heads (CH) which disseminated in the area and behave as well as Camera Nodes (CN). These nodes will require higher capability than Mica2 Motes. So, we set the parameters of these nodes to behave as normal MANET nodes. The Cluster Heads $(\mathrm{CH})$ nodes will aggregate the simple scalar data transmitted periodically by Mica2 Motes and forward it to the data sink node, through the two access point nodes.

The Camera Nodes (CN) will start sending randomly five seconds of video to simulate an intrusion detection event and forward it to the requesting sink nodes through other $\mathrm{CH} /$ Video sensor nodes. The video data from any $\mathrm{CH} / \mathrm{CN}$ can be made available to any of the five video sink (VS) nodes. In this scenario, we can increase the simultaneous video connections between the visual sensor nodes and the video sinks from 1 to 5 and study how the "Normal sensor DATA" is getting effected by the video traffic. 
B. The Simulation of Video Traffic

Based on the traffic model proposed in [3], the packet generation sequence is controlled, as shown in the following diagram.

The output of the computational steps are performed as follows:

1. Transmission of a video packet when video traffic starts.

2. Compute the transmission time of the next video packet (Next Video Packet Time) NVPT=lognormal $\left(\mathrm{x}_{1}, \mathrm{y}_{1}\right)$

3. Computation of time $t_{i}$ needed in order the next video packet to be transmitted $t_{i=} \operatorname{lognormal}\left(\mathrm{x}_{2}, \mathrm{y}_{2}\right)$

4. Different parameters are used to calculate inter-video packet time (variable NVPT), in order to take in consideration different packet sizes, and the inter-control packet time (array $t_{i}$ ). The values of $t_{1}$ to $t_{n}$ are summed to variable SumNVPT (SumNVPT $=t_{1+} t_{2+} t_{3+} t_{4+} t_{5+\ldots \ldots+} t_{n}$ ).

5. While the value of SumNVPT is less than NVPT, $t_{i}$ is used as the inter-packet time for transmission of small packets. In any other case, the inter- packet time is NVPT- (SumNVPT - $\left.t_{i}\right)$.

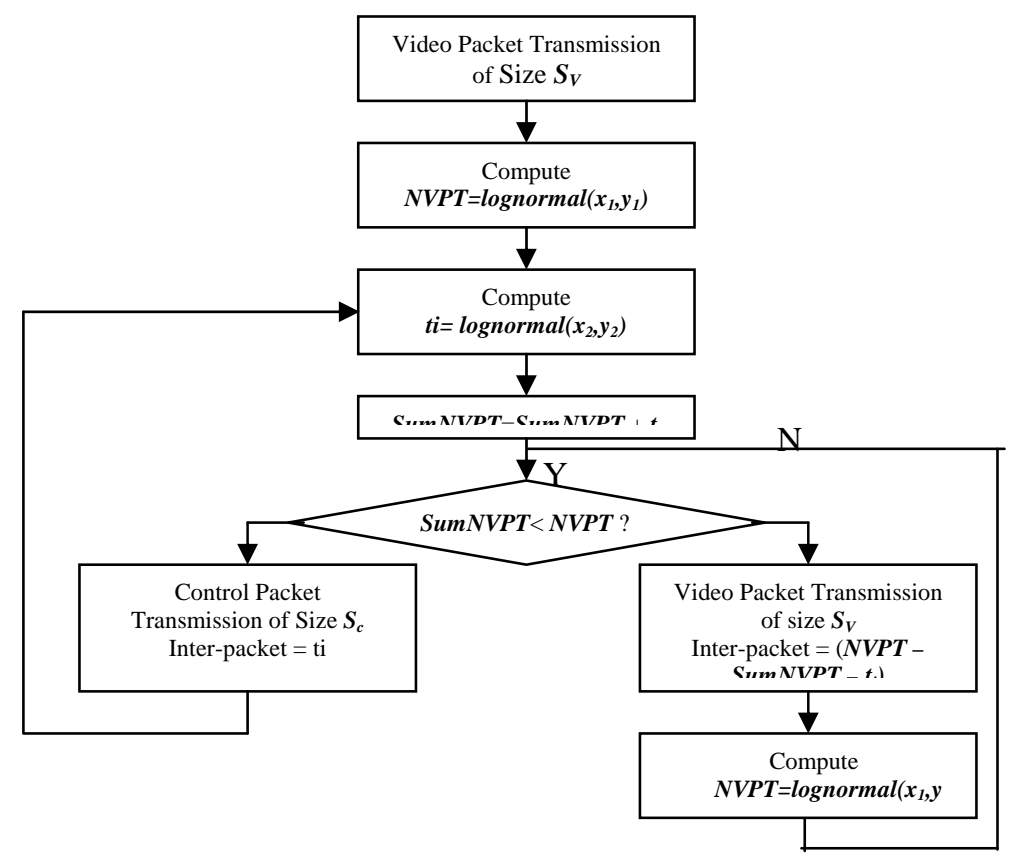

Fig. 1. The video traffic generation model.

The size of control packets SC is assuming as 130 bytes and the size of video packets SV is 8015 bytes. Moreover, in this scenario the log-normal distribution with the parameters x1 equals 1.5514 and y1 equals 3.7143 and is used to simulate the inter-video packet time interval for big packets while the log-normal distribution with parameters x2 equals to 2.5647 and y2 equals to -9.1022 and is used to simulate the interpacket time for small packets. Looking the traffic pattern of the original and the simulated video traffic from work of Peng Gao et all [28], it is clear that the simulated traffic model almost resembles the original traffic pattern. Moreover, in that work [28], the traffic generator has been tested on a high bandwidth wired network simulation model. In this approach, the video traffic generator on wireless video sensor network with some lower capability wireless nodes is being tested.

\section{About the Mica2 Motes [16] Sensor Node’s Data Generation}

The Mannasim [18],[19] has a simulation mechanism for simple scalar data. In the following table (Table 2) there is a brief description of the parameters that used from Mannasim in order the Mica2 Motes to be simulated. The Mannasim Framework provides temperature and carbon monoxide data generators.

Table 2. Mannasim Framework for Mica2 Motes Sensor Node’s Data Generation

\begin{tabular}{|l|l|}
\hline \multicolumn{1}{|c|}{ Parameter } & \multicolumn{1}{c|}{ Description } \\
\hline Data Generator Type & Synthetic data should be produced during simulation time \\
\hline Data Average Value & The average deviation of the used Gaussian distribution \\
\hline Data Standard Deviation & The standard deviation of the used Gaussian distribution \\
\hline Maximum Data Value & Maximum allowed value \\
\hline
\end{tabular}




\section{SIMULATION RESULTS}

A. The Parameters of the Simulation

\begin{tabular}{|c|c|}
\hline Basic Parameters & \\
\hline Channel & $\begin{array}{l}\text { Wireless } \\
\text { Channel }\end{array}$ \\
\hline Propagation Model & $\begin{array}{l}\text { TwoRayGroun } \\
\text { d }\end{array}$ \\
\hline Physical Model & WirelessPhy \\
\hline Mac Protocol & 802_11 \\
\hline QueueType & $\begin{array}{l}\text { DropTail } \\
\text { PriQueue }\end{array}$ \\
\hline Queue Length (number of packets) & 300 \\
\hline Antenna Type & Omni Antenna \\
\hline Energy Model & Battery \\
\hline \multicolumn{2}{|l|}{ Traffic parameters } \\
\hline Video Transport Agent Type & UDP \\
\hline Sensor Data Transport Agent Type & UDP \\
\hline Routing Protocol & AODV \\
\hline Common Node Disseminating Type & 0 (Continuous) \\
\hline CH Node Disseminating Type & 0 (Continuous) \\
\hline Mica2Motes Disseminating Interval & $5 \mathrm{sec}$ \\
\hline VS/CH Disseminating Interval & $10 \mathrm{sec}$ \\
\hline Sensor Data Port & 2020 \\
\hline Video Port & 2222 \\
\hline Video Data Start & Every $5^{\text {th }}$ sec \\
\hline Video Data Duration & $5 \mathrm{sec}$ \\
\hline Video Data Simulation Interval & $20 \mathrm{sec}$ \\
\hline Number of Simultaneous Connections & $1,2,3,4$ \\
\hline \multicolumn{2}{|c|}{ Mica2 Motes parameters (Atmel 128L 8MHZ - 8MIPS) } \\
\hline Transmission Range & $50-100 \mathrm{~m}$ \\
\hline Initial Energy & 10 Joules \\
\hline Bandwidth & $28.8 \mathrm{kbps}$ \\
\hline Sensing Power & $0.015 \mathrm{~W}$ \\
\hline Processing Power & $0.024 \mathrm{w}$ \\
\hline Instructions per Second & 80000000 \\
\hline \multicolumn{2}{|c|}{ VS-Node parameters (Intel Strong Arm 1100133 MHZ --> 150 MIPS) } \\
\hline Transmission Range & $250-300 \mathrm{~m}$ \\
\hline Initial Energy & 100 Joules \\
\hline VS sensing Power & $0.064 \mathrm{~W}$ \\
\hline VS processing Power & $0.360 \mathrm{~W}$ \\
\hline Instructions per Second & 150000000 \\
\hline
\end{tabular}

In this simulation the image processing and storage aspects of the visual sensor nodes were not taken into account since we only focus on the transport layer and network layer issues of the VSN design. 


\section{B. Metrics Used for Evaluation}

The impact of increasing the number of simultaneous video connections on normal sensor data flow as well as the video traffic flow to the video sink nodes is studied in this work. Therefore, the performance/overhead of Mica2 Motes and visual sensor nodes are measured.

The event trace file of a Mannasim simulation will be entirely different from normal NS-2 event trace file. This is because, not all the data packets generated at Mica2 Motes are actually sent to the data sink node, under Mannasim. Because, the data aggregated by the cluster head node will actually sent to the data sink. In fact, the packets sequence numbers generated at the Mica2 motes are entirely different from those arriving at the data sink. Because, the cluster head nodes will aggregate the data and generate new packets with different sequence numbers. The metrics applied to perform the impact analysis are summarized in the following:

1) Data Packets Received

The number of data packets received at sink node is considered as an important metric to study the impact of video traffic on normal sensor data flows.

2) Normalized Routing Load

Normalized routing load is the ratio between the number of routing packets generated and the number of data packets successfully delivered.

Normalized Routing Load at Mica2 Motes is measured as the ratio between the number of routing packets generated and data packets successfully delivered at data sink.

Normalized Routing Load at Visual Sensors is measured as the ratio between the number of routing packets generated and video packets successfully delivered at video sink.

3) Routing Message Overhead

In this work we measure Overhead in terms of number of generated/forwarded routing messages. We measure them separately at Mica2 Motes as well as VS-Nodes.

4) Dropped Packets at Mica2 Motes

The number of Dropped packets in a WSN/VSN scenario is an important metric to evaluate the network performance. Generally, the packets will be dropped by several reasons in a wireless ad hoc network scenario. We count the Packets Dropped at Mica2 Motes and VS-Nodes in order to understand the dropping behavior at two different levels.

5) Throughput of Scalar Data Flow

Normalized throughput is a ratio between the number of data packets successfully delivered and the duration of traffic. It is generally measured in Kbps/Mbps.

We measure throughput of video flow as the ratio between the number of video packets successfully delivered and the duration of overall video traffic. We measure throughput of data flow at Mica2 Motes as the ratio between the number of scalar data packets successfully delivered and the duration of overall data traffic.

6) MAC Load

The MAC load is the ratio between the number of packets sent at MAC layer and the number of data packets successfully delivered.

The MAC Load at Mica2 Motes is measured as the ratio between the packets sent at MAC layer of Mica Motes and data packets successfully received at data sink.

The MAC Load at VS-Nodes is measured as the ratio between the packets sent at MAC layer of Visual Sensor nodes and video packets successfully received at video sink.

\section{7) Average Consumed Energy}

The average consumed energy is the average of energy spent by all the nodes in the network and is measured in Joules. In this analysis we separately calculate Average Consumed Energy by Mica2 Motes as well as VSNodes to understand the energy consumption behaviors of the two types of nodes.

8) Packet Delivery Fraction (PDF)

The PDF is the ratio between the number of generated data at source and the number of data packets successfully received at sink. The PDF of data flow is not considered because the data generated by the Mica2 Motes sources will actually not reach the destination. So, only the PDF of Video flows are considered. This is measured as the ratio between the number of generated video packets at visual sensor nodes and the number of video packets successfully received at video sink.

9) Average End to End Delay (EED) of Video Packets (ms)

To measure the End to End Delay, the time in which the packet is generated and the time in which the same packet arrived at the sink were needed. The sequence number of the packet is used to identify each packet during this calculation. Since the sequence number of sent packets at Mica2 Motes and received packets at data 
sink will be different in a Mannasim simulation, we didn’t consider finding EED of normal sensor data flows (Mannasim directly display the EED of each data packet during the simulation on the console itself).

So, only the End to End Delay of video traffic is measured herein. The EED of Video Packets is the time in which the video packet is generated at source and the time in which the same packet is arrived at the video sink.

C. The Results

The following table shows the measurements made on the Visual Sensor Network's Video flows and normal data flows. The values shown in the table are the average of three runs of the simulation. The first table shows the results measured for analyzing the flows between the Mica2 Motes and the data sink node. The second table shows the results measured for analyzing the flows between the Mica2 Motes and the data sink node.

Table 3. Measurements Made on Visual Sensor Network

\begin{tabular}{|c|c|c|c|c|c|c|c|}
\hline $\begin{array}{c}\text { Number of } \\
\text { Video } \\
\text { Connections }\end{array}$ & $\begin{array}{c}\text { Scalar Data } \\
\text { Packets } \\
\text { Received at } \\
\text { Data Sink }\end{array}$ & $\begin{array}{c}\text { Normalized } \\
\text { Routing } \\
\text { Load }\end{array}$ & $\begin{array}{c}\text { Overhea } \\
\mathrm{d}\end{array}$ & $\begin{array}{c}\text { Dropped } \\
\text { Packets }\end{array}$ & $\begin{array}{c}\text { Throughput } \\
\text { of Scalar } \\
\text { Data Flow } \\
\text { (kbps) }\end{array}$ & $\begin{array}{c}\text { MAC } \\
\text { Load }\end{array}$ & $\begin{array}{c}\text { Average } \\
\text { Consumed } \\
\text { Energy (Joules) }\end{array}$ \\
\hline 1 & 60 & 22.69 & 4084 & 1288 & 0.23 & 55.42 & 0.9 \\
\hline 2 & 35 & 44.98 & 4722.67 & 1255 & 0.13 & 114.43 & 1.17 \\
\hline 3 & 20.33 & 99.03 & 6041 & 1927 & 0.09 & 197.67 & 1.19 \\
\hline 4 & 20.33 & 92.97 & 5671.33 & 1767.67 & 0.08 & 222.95 & 1.29 \\
\hline
\end{tabular}

With respect to the Mica2 Motes and their Data flows (While using TCP as the Transport Protocol for Video)

Table 4. Measurements Made on Visual Sensor Network

\begin{tabular}{|c|c|c|c|c|c|c|c|}
\hline $\begin{array}{c}\text { Number of } \\
\text { Video } \\
\text { Connections }\end{array}$ & $\begin{array}{c}\text { Scalar Data } \\
\text { Packets } \\
\text { Received at } \\
\text { Data Sink }\end{array}$ & $\begin{array}{c}\text { Normalized } \\
\text { Routing } \\
\text { Load }\end{array}$ & $\begin{array}{c}\text { Overhea } \\
\mathrm{d}\end{array}$ & $\begin{array}{c}\text { Dropped } \\
\text { Packets }\end{array}$ & $\begin{array}{c}\text { Throughput } \\
\text { of Scalar } \\
\text { Data Flow } \\
\text { (kbps) }\end{array}$ & $\begin{array}{c}\text { MAC } \\
\text { Load }\end{array}$ & $\begin{array}{c}\text { Average } \\
\text { Consumed } \\
\text { Energy (Joules) }\end{array}$ \\
\hline 1 & 41 & 16.38 & 2015 & 761.33 & 0.15 & 42.45 & 0.8 \\
\hline 2 & 30.33 & 33.55 & 3052.67 & 954 & 0.09 & 90.99 & 1.44 \\
\hline 3 & 23 & 44.56 & 3074.33 & 1211.32 & 0.07 & 121.21 & 1.39 \\
\hline 4 & 22.33 & 51.44 & 3446.66 & 1529.66 & 0.06 & 134.43 & 1.48 \\
\hline
\end{tabular}

With respect to the Mica2 Motes and their Data flows (While using UDP as the Transport Protocol for Video)

Table 5. Measurements Made on Visual Sensor Network

\begin{tabular}{|c|c|c|c|c|c|c|c|c|}
\hline $\begin{array}{c}\text { Number of } \\
\text { Video } \\
\text { Connections }\end{array}$ & $\begin{array}{c}\text { PDF of } \\
\text { Video } \\
\text { Flows }\end{array}$ & $\begin{array}{c}\text { Normalized } \\
\text { Routing } \\
\text { Load }\end{array}$ & Overhead & $\begin{array}{c}\text { Dropped } \\
\text { Packets }\end{array}$ & $\begin{array}{c}\text { Throughput } \\
\text { of Video } \\
\text { Flow (kbps) }\end{array}$ & $\begin{array}{c}\text { MAC } \\
\text { Load }\end{array}$ & $\begin{array}{c}\text { Average } \\
\text { EED of } \\
\text { Video } \\
\text { Packets } \\
\text { (ms) }\end{array}$ & $\begin{array}{c}\text { Average } \\
\text { Consumed } \\
\text { Energy } \\
\text { (Joules) }\end{array}$ \\
\hline 1 & 32.59 & 0.90 & 462.00 & 1106.67 & 97.08 & 4.17 & 93.52 & 1.33 \\
\hline 2 & 32.89 & 1.17 & 388.33 & 972.33 & 131.82 & 4.00 & 158.58 & 1.77 \\
\hline 3 & 31.61 & 1.19 & 906.33 & 2056.33 & 122.94 & 4.66 & 106.44 & 1.79 \\
\hline 4 & 31.96 & 1.29 & 657.33 & 1643.33 & 139.17 & 4.19 & 174.06 & 1.91 \\
\hline
\end{tabular}

With respect to the Visual Sensor Nodes and their Data flows (While using TCP as the Transport Protocol for Video) 
Table 6. Measurements Made on Visual Sensor Network

\begin{tabular}{|c|c|c|c|c|c|c|c|c|}
\hline $\begin{array}{c}\text { Number } \\
\text { of } \\
\text { Video } \\
\text { Connect } \\
\text { ions }\end{array}$ & $\begin{array}{c}\text { PDF } \\
\text { of } \\
\text { Video } \\
\text { Flows }\end{array}$ & $\begin{array}{c}\text { Normalized } \\
\text { Routing } \\
\text { Load }\end{array}$ & Overhead & $\begin{array}{c}\text { Dropped } \\
\text { Packets }\end{array}$ & $\begin{array}{c}\text { Throughput } \\
\text { of Video } \\
\text { Flow (kbps) }\end{array}$ & $\begin{array}{c}\text { MAC } \\
\text { Load }\end{array}$ & $\begin{array}{c}\text { Average } \\
\text { EED of } \\
\text { Video } \\
\text { Packets } \\
\text { (ms) }\end{array}$ & $\begin{array}{c}\text { Average } \\
\text { Consumed } \\
\text { Energy } \\
\text { (Joules) }\end{array}$ \\
\hline 1 & 20.2 & 0.8 & 99.67 & 1223.33 & 92.3 & 2.59 & 1313.55 & 1.18 \\
\hline 2 & 16.41 & 1.44 & 173.67 & 2801.33 & 158.75 & 2.77 & 3688.05 & 2.13 \\
\hline 3 & 12.24 & 1.39 & 222.33 & 4116 & 144.39 & 3.04 & 3797.39 & 2.02 \\
\hline 4 & 8.89 & 1.48 & 379 & 6627.66 & 155.06 & 3.1 & 4891.75 & 2.16 \\
\hline
\end{tabular}

With respect to the Visual Sensor Nodes and their Data flows (While using UDP as the Transport Protocol for Video)

D. The Analysis on Results

In the following two line graphs, we compare the dropped packets with respect to the increase of number of simultaneous video connections. As shown in the following graphs (Fig. 2a, 2b) in terms of dropped packets, the mica motes are getting affected very much with respect to the increase in number of simultaneous video connections, irrespective of the type of transport agent used for sending video traffic. But if we closely note these two graphs, the use of TCP indirectly causes much packet drop at mica motes. But while using TCP, the packet drops at visual sensor nodes (VS-Nodes) is getting reduced by almost $35 \%$ (33.8 \%).

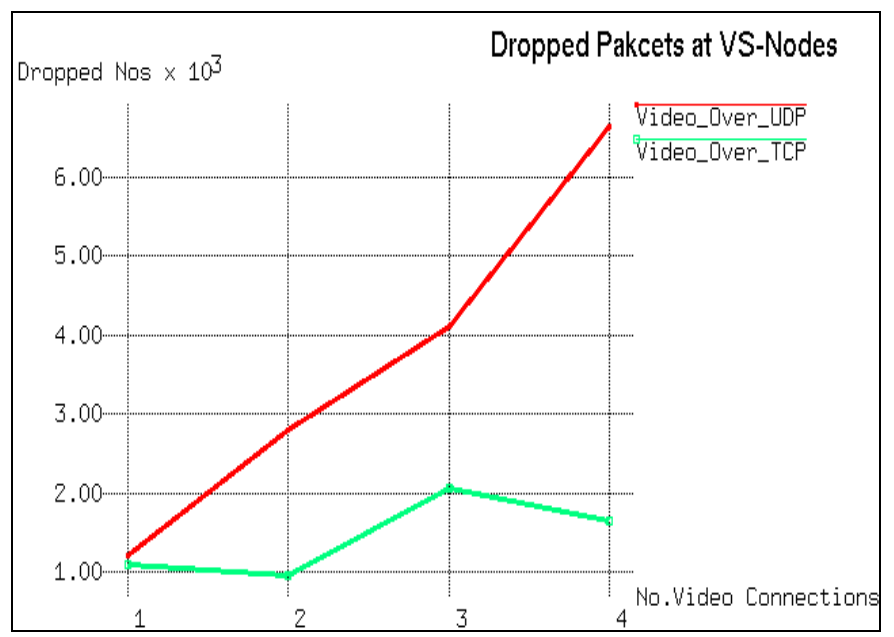

Fig. 2a. Number of Dropped Packets at Visual Sensors

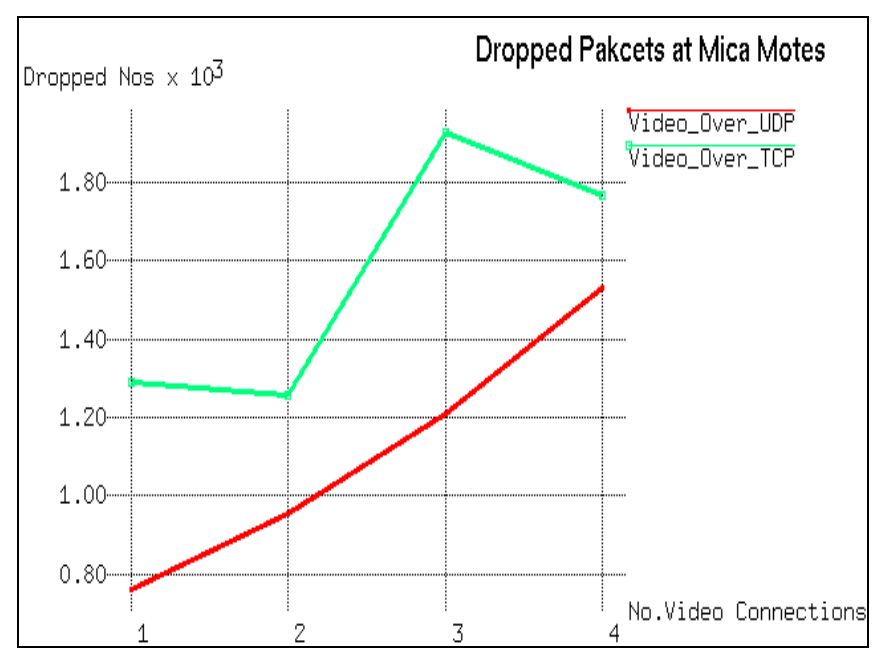

Fig. 2b. Number of Dropped Packets at Mica Motes 
The following two line graphs (Fig.s 3a, 3b) shows the overhead with respect to the increase of number of simultaneous video connections. Here we measure overhead in terms of total number of routing messages generated and forwarded from the mica motes and video sensor nodes. As shown in the line graph, the overhead at mica motes (denoted as WSN) is getting affected very much with respect to the increase in number of simultaneous video connections while using TCP for video flows.

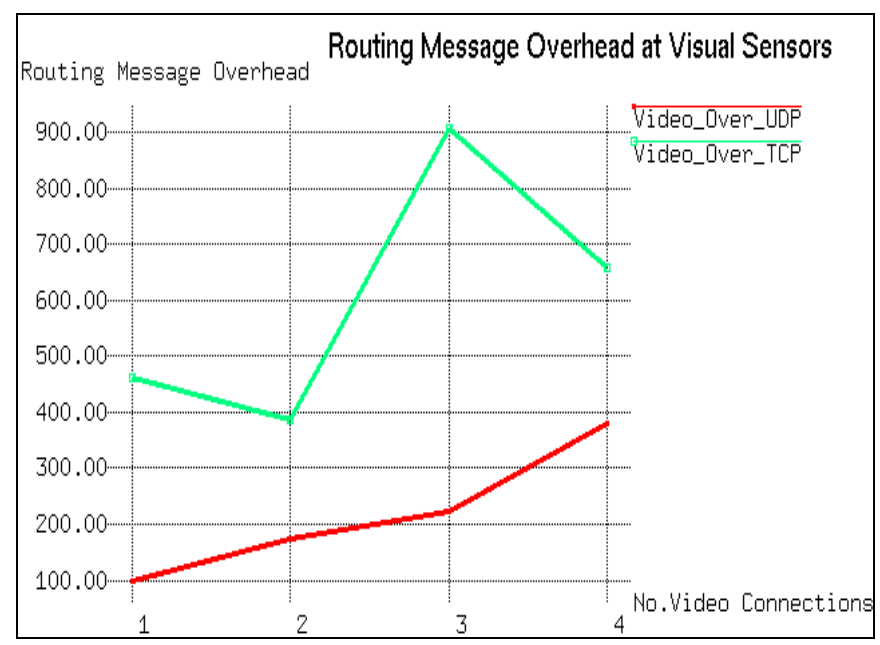

Fig. 3a. Overhead at Visual Sensors

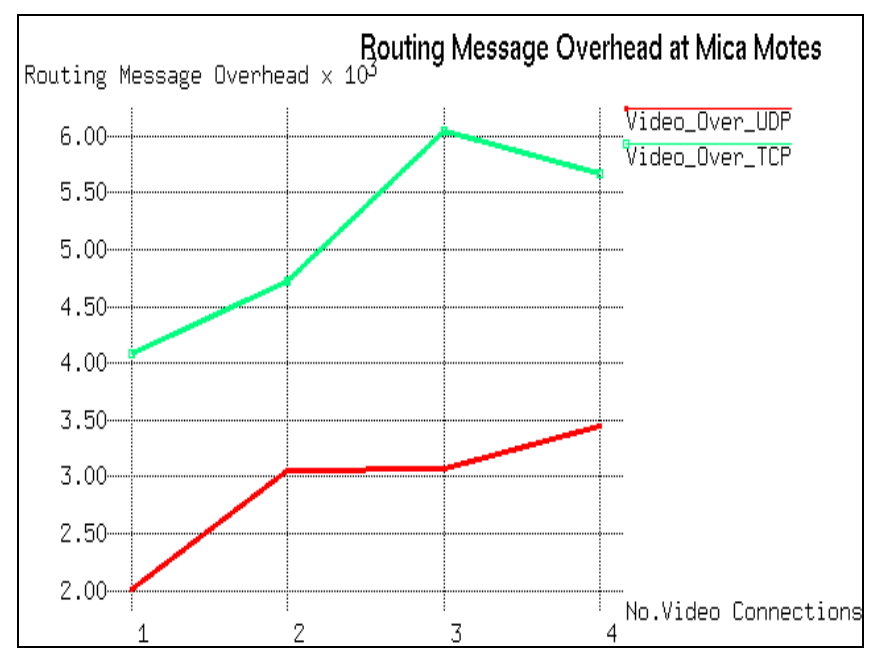

Fig. 3b. Overhead at Mica Motes

The following two line graphs (Fig. 4a, 4b) shows the energy consumption with respect to the increase of number of simultaneous video connections. Here we measure Energy consumption in Joules. As shown in the line graph, the energy consumption of mica motes as well as the visual sensor nodes are increasing with respect to the increase in number of simultaneous video connections. Since the Visual Sensor nodes process much data, obviously, it is consuming much energy than the mica motes. But, while using TCP for video traffic, then it reduces the energy consumption very much. 


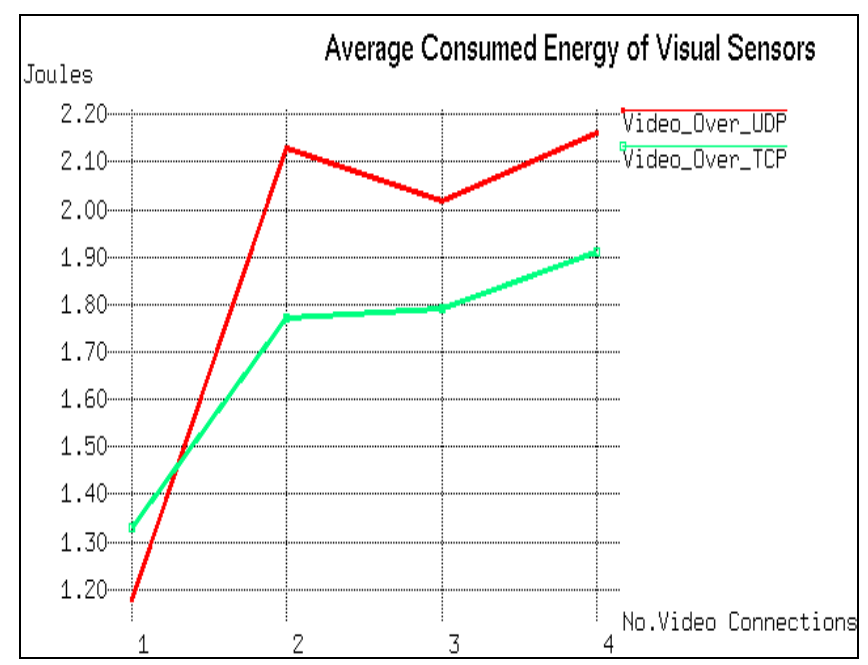

Fig. 4a. Average Consumed Energy at Visual Sensors

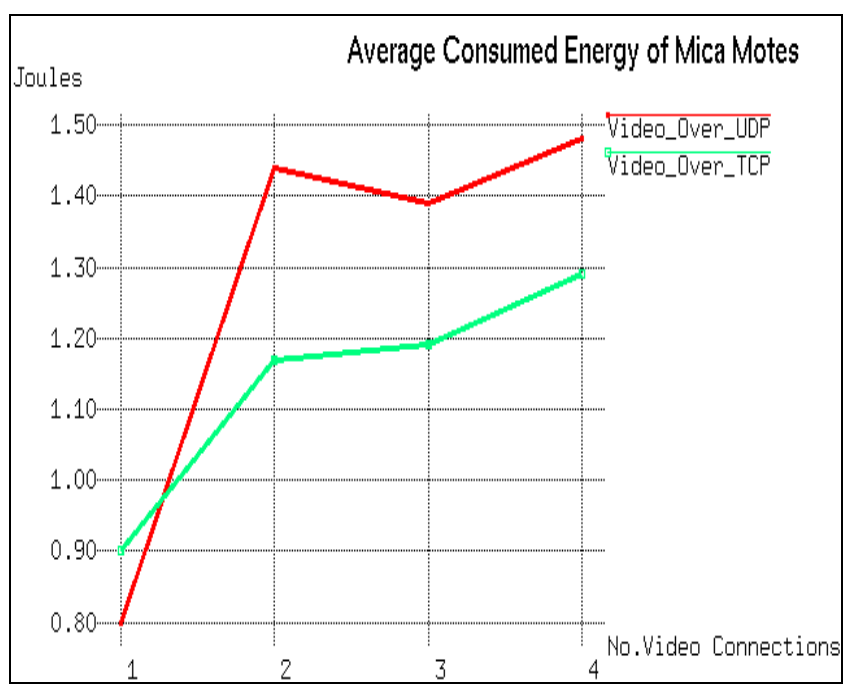

Fig. 4b. Average Consumed Energy at Visual Sensors

The following two line graphs (Fig. 5a, 5b) shows the MAC Load with respect to the increase of number of simultaneous video connections MAC load is increasing with respect with respect to the increase in number of simultaneous video connections.

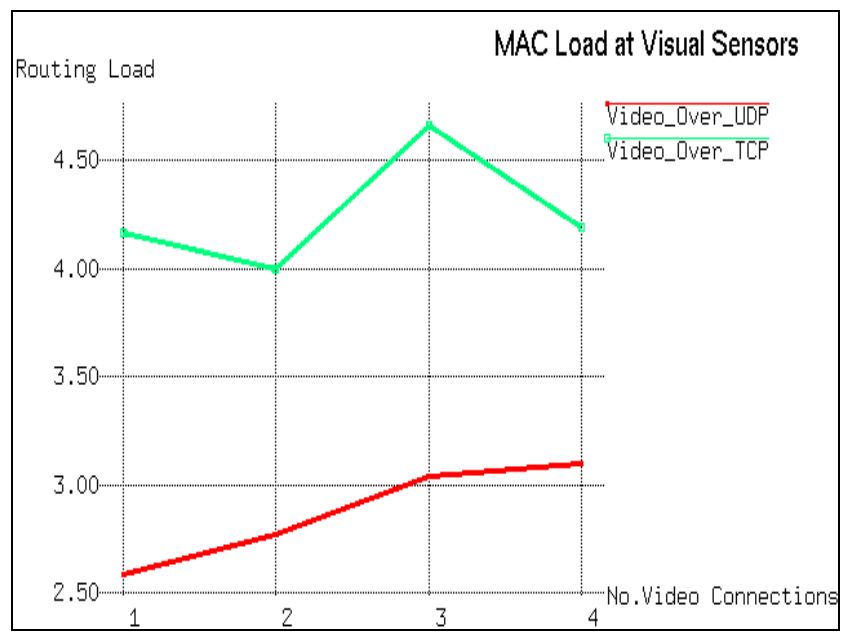

Fig. 5a. MAC Load at Visual Sensors 


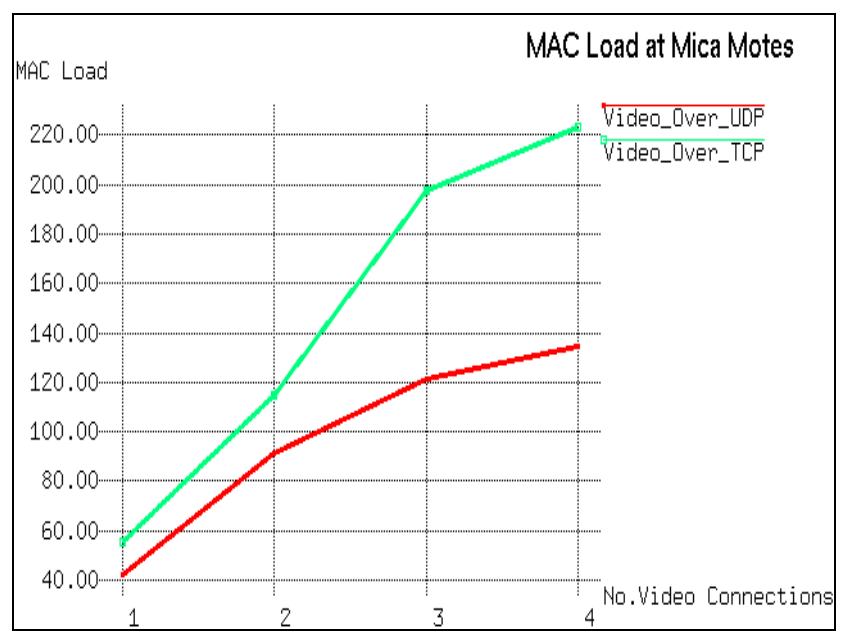

Fig. 5b. MAC Load at Mica Motes

The following two line graphs (Fig. 6a, 6b) shows the routing load with respect to the increase of number of simultaneous video connections routing load is increasing with respect with respect to the increase in number of simultaneous video connections. But, the routing load at visual sensor nodes was minimum while using TCP for video transport. But the routing load at the mica motes was high while using TCP for video transport

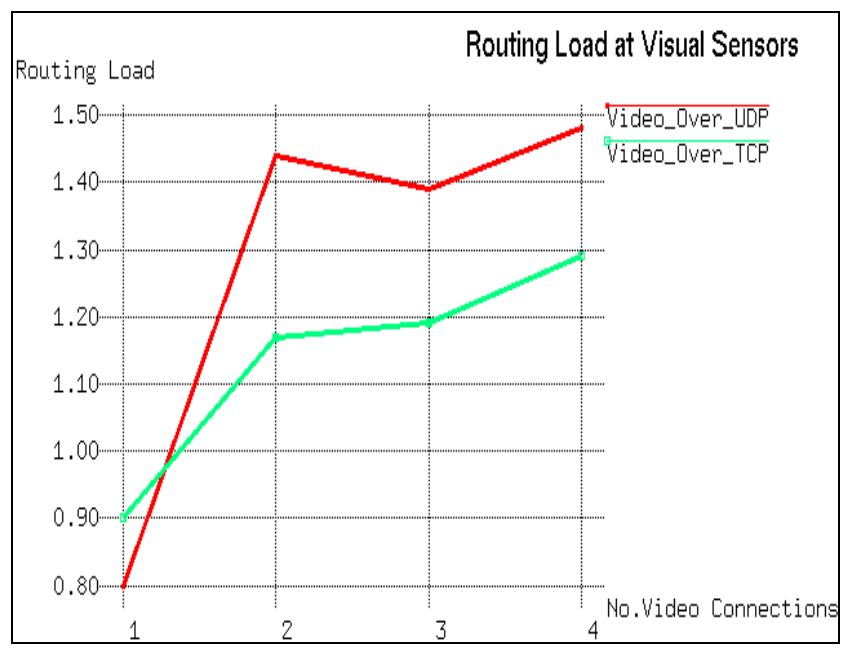

Fig. 6a. Routing Load at Visual Sensors

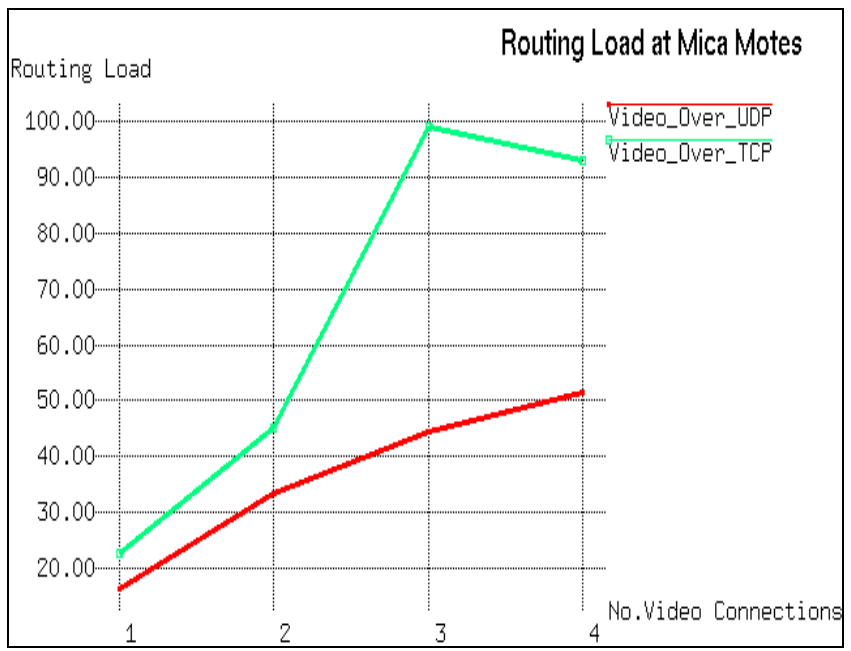

Fig. 6b. Routing Load at Mica Motes 
The following graph (Fig. 7) shows the Data Packets Received at Sink at different video load on the network. The increase in number of simultaneous video connection obviously affects the number of scalar data packets reaching the data sink which were sent from the mica motes. But, while using TCP for video, then it improves the performance a little bit in some cases. Basically, the end to end delay of data packets in a typical sensor network will be high because of the delays involved in data dissemination and aggregation.

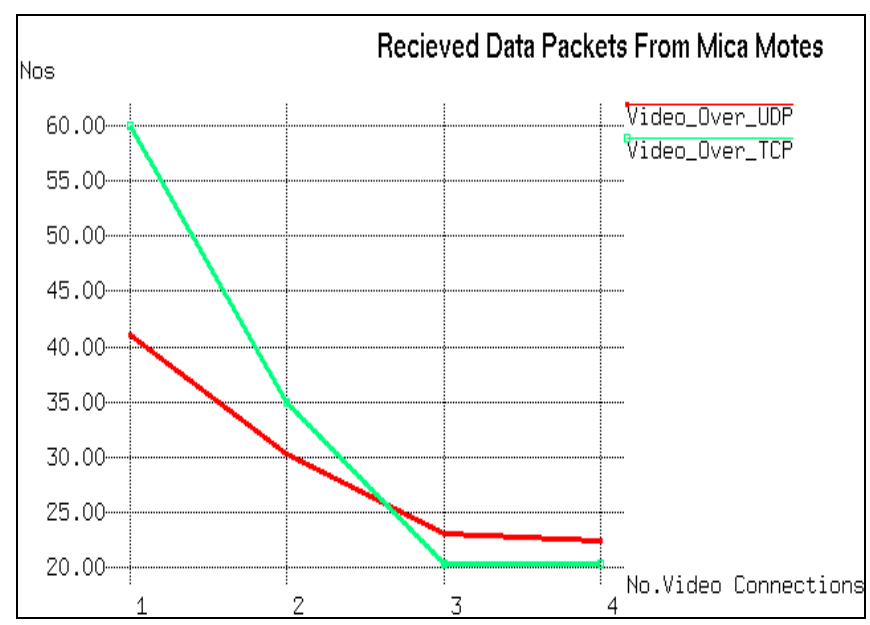

Fig. 7. The Analysis on Sensor Data Packets Received at Sink

The following graph (Fig. 8) shows the average end to end delay of video packets at different video load on the network. The increase in number of simultaneous video connection obviously considerably increase the end to end delay while using UDP transport for video. But while using TCP for video transport the average end to end delay was much better and almost at same level even for different number of simultaneous video connections. Because of the high end to end delay is a big obstacle in using this network for real-time monitoring application.

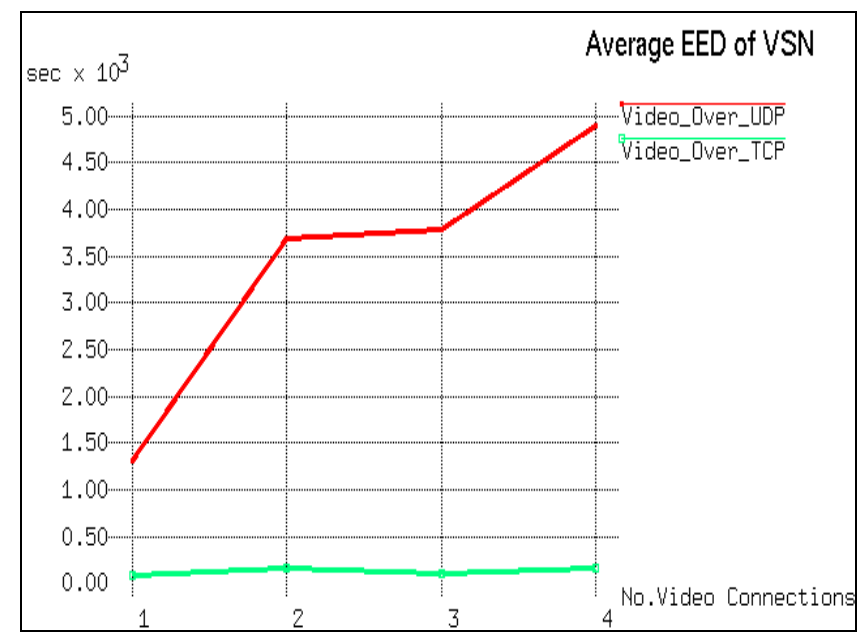

Fig. 8. The Average End to End Delay of Video Packets

The following graph (Fig. 9) shows the average packet delivery fraction/ratio of video packets at different video load on the network. The increase in number of simultaneous video connection rapidly decrease the pdf. But while using, TCP for video transport, then the pdf was comparatively good. The low pdf makes impossible to use this network for sending high quality graphics and using conventional encoding schemes. To overcome this problem, the video coding schemes should be designed in such a way to with stand high packet loss. 


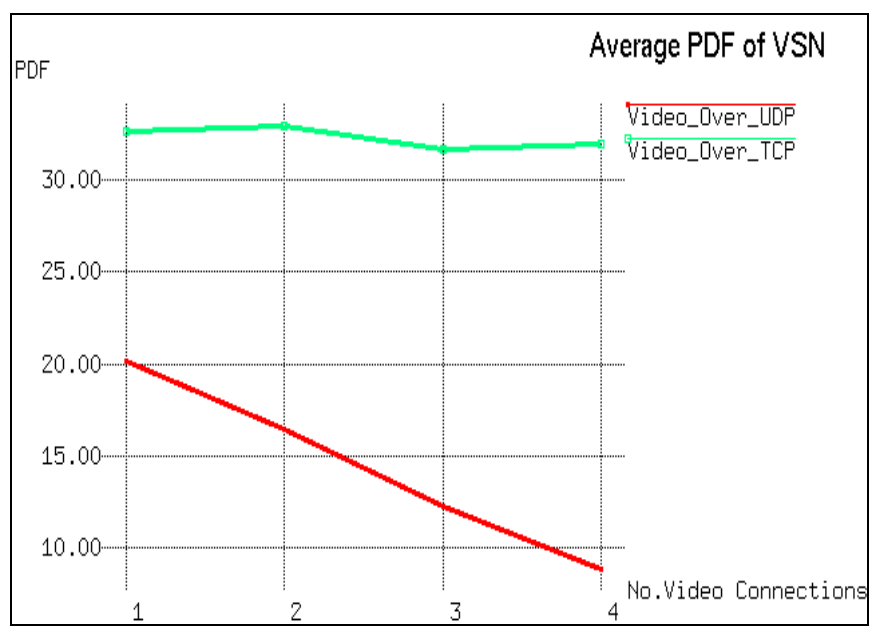

Fig. 9. The Average PDF of VSN

The following two graphs (Fig. 10a, 10b) shows the throughput of video traffic as well as the normal sensor data flow at different video load on the network. As shown in the first graph (Fig. 10a), the throughput was increasing with respect to the increase of connections. But, while using TCP, for video transport then the throughput is increasing for data flow and decreasing for the video flow.

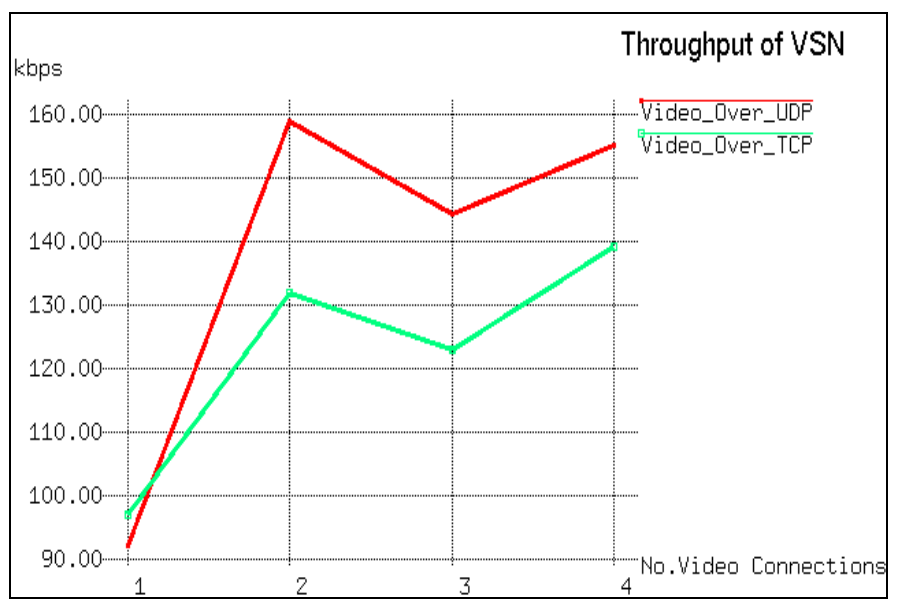

Fig. 10a. Throughput on Visual Sensors

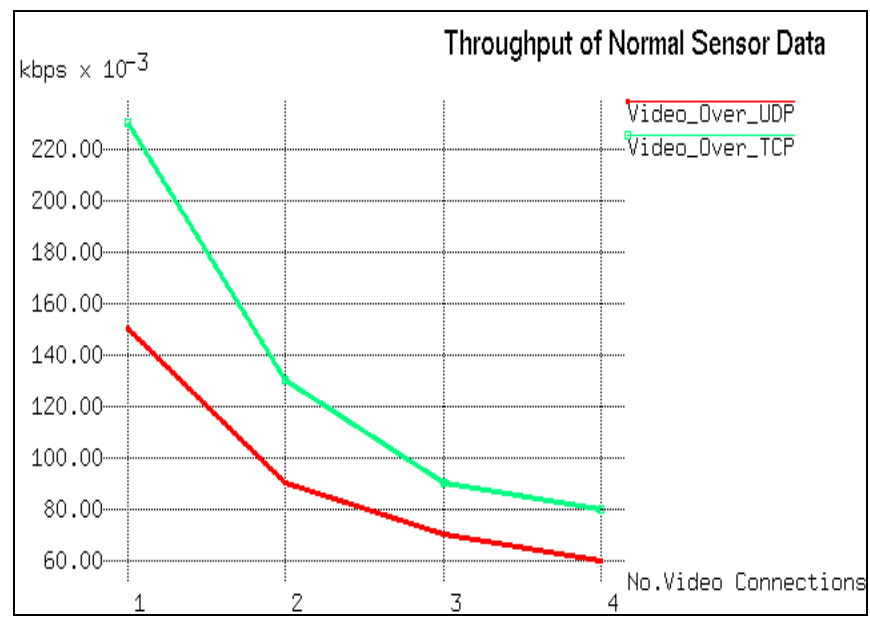

Fig. 10b. Throughput on Mica Motes

E. Problems Identified and Suggestion for Improvements

In this VSN network, the normal Mica2 Motes and the visual sensor nodes uses same communication channel since the same visual sensor node will also act as the cluster head for aggregating the scalar sensor data sent by the mica motes. Since we use the same channel for transmitting video packets from the visual sensor nodes, it is interfering with the communication of mica motes and affects their performance. Similarly, there are lot of 
simple Mica2 Motes sensor nodes in the network, and they will try to send packets periodically and affect the video communication of visual sensor nodes. If it is possible to use different communication channels for the two different flows (data and video) then it will improve the performance considerably.

The low pdf, high packet dropping ratio and low throughput makes it hard to use this simple network for sending high quality graphics and using conventional encoding schemes. It shows that only low resolution and low rate video is possible under this lower capability VSN. At the sink, it will not be possible to reconstruct a meaningful video sequence from the received frames/packets which are encode in conventional encoding schemes. To improve this, we may design a custom, low rate video encoding scheme which should withstand against high packet loss and low throughput.

In this experiment, we used the IEEE 802.11 as the MAC layer protocol. If we design the visual sensor nodes as much powerful nodes that can handle high bandwidth using more advance MAC layer protocol, then we can improve the overall performance.

Further, the Mica Motes of Mannasim also uses AODV, even though they generally will not forward packets more than one hop. This happens due to the fact that the scalar sensor data will be forwarded to data sink through application level forwarding mechanism only using one hop broadcast to a specific application port. It may also be a reason for the poor performance. We may avoid this in future design by just using a one hop broadcast without any routing agent to improve the performance.

In Visual sensor nodes, we used AODV as routing protocol since the video flows will need multi hop routing to reach remote video sinks. But, if we assume the VSN scenario as a static network of nodes without any mobility, then the periodic broadcast in AODV produce also an unnecessary overhead. Because, if nodes are not moving, then there will not be any necessity for very frequent route discovery. So we may modify the route discovery mechanism of AODV and suit it for the static visual sensor network. This will also improve the preference to a considerable level.

The use of TCP protocol for video transport improved the performance in most of the cases.

\section{CONCLUSION}

Adding visual sensor nodes or camera nodes in an existing sensor network definitely affects the overall performance of the sensor network. The results from the previous section clearly proves the performance reduction. Increasing the simultaneous video flows just affects all the aspects of the network. Moreover, the outcome of this research justify that the hardware meant for normal sensor network will only support simple scalar data flows. The IEEE 802_11 MAC protocol and the common UDP transport protocol which are generally used in sensor network are also not sufficient to handle simultaneous video flows of a VSN. TCP performed better than UDP while handling video traffic. The simple network such as the one presented in this paper can only support visual sensor data such as low quality images. Using them for sending video with its present hardware capability is not possible.

The communication overhead should be reduced to improve the performance of both data flows and video flows. For that we may consider using much powerful visual sensor nodes with advance transport layer, network layer, and physical layer protocols. In the previous section we highlighted some of the problems identified during the simulation and provided some suggestion for improvements in video encoding and routing protocols. That also can be considered in future design of VSN protocols.

In this work the issues of the typical visual sensor nodes such as image processing, storage and camera calibration were not taken in to account since NS-2/Mannasim were not at all having facilities to incorporate those. Further we only concentrate on the transport layer and network layer issues of the VSN design. So future works may incorporate missing issues of VSN also in their network/simulation design.

\section{REFERENCES}

[1] Albano M., Chessa S., Di Pietro R., “Information Assurance in Critical Infrastructures via Wireless Sensor Networks”, IAS 2008, Proceedings of The Fourth International Conference on Information Assurance and Security, Sept. 8-10, (Napoli) Italy, 2008

[2] Akylidiz I. F., Su W., Sankarasubramaniam Y., Cayirci E., “A survey on sensor networks”, "IEEE Personal Communications Magazine”, Vol. 40 Iss. 8, pp. 102-114, 2002

[3] Kanakaris V., Ndzi D., Ovaliadis K., “Applications of MANET routing protocols in sensor network”, "International Journal of Research and Reviews in Ad Hoc Networks (IJRRAN)”, Vol. 1, Iss. 4, pp. 88-92, Dec. 2011

[4] Hadjidj A., Souil M., Bouabdallah A., Challal Y., Owen H., "Wireless sensor networks for rehabilitation applications: Challenges and opportunities Review Article”, “Journal of Network and Computer Applications”, Vol. 36, Iss. 1, pp. 1-15, Jan. 2013.

[5] Akyildiz I. F., Melodia T., Chowdhury K. R., “A survey on wireless multimedia sensor networks”, "Computer Networks”, Vol. 51, pp. 921-960, 2007

[6] Soro S., Heinzelman W., “A survey of visual sensor networks”, “Advances of Multimedia”, 2009.

[7] Almalkawi I., Zapata M., Al-Karaki J., Julian Morillo-Pozo J., "Wireless Multimedia Sensor Networks: Current Trends and Future Directions”, “Sensors”, Vol. 10, Iss. 7, pp. 6662-6717, Jul. 2010.

[8] Hengstler S., Prashanth D., Fong S., Aghajan H., "Mesh-Eye: a hybrid-resolution smart camera mote for applications in distributed intelligent surveillance," in Proceedings of the 6th International Symposium on Information Processing in Sensor Networks (IPSN '07), pp. 360-369, 2007.

[9] Akyildiz I. F., Su W., Sankarasubramaniam Y., Cayirci E., “Wireless sensor networks: a survey”, “Computer Networks (Elsevier) Journal”, Vol.38, No. 4, pp. 393-422, March 2002. 
[10] Akyildiz I. F., Vuran M. C., Akan O. B., Su W., “Wireless Sensor Networks: A Survey Revisited,” to appear in Computer Networks Journal (Elsevier), 2005.

[11] Eren G., Özgür A. "Multimedia Communication in Wireless Sensor Networks”, “Annals of Telecommunications”, Vol. 60, Iss. 7-8, pp. 872-900, August 2005.

[12] Misra S., Reisslein M., Xue G., "A survey of multimedia streaming in wireless sensor networks", IEEE Communications Surveys and Tutorials, Vol. 10, pp. 18-39, 2008.

[13] Charfi Y., Wakamiya N., Murata M., "Challenging issues in visual sensor networks," Tech. Rep., Advanced Network Architecture Laboratory, Osaka University, 2007.

[14] Costa DG, Silva I, Guedes LA, Vasques F, Portugal P., “Availability issues in wireless visual sensor networks.”, "Sensors (Basel)”. Vol. 14. Iss. 2, February 2014.

[15] Tavli B., Bicakci K., Zilan R., Jose M. Barcelo-Ordinas, “A survey of visual sensor network platforms”, "Multimedia Tools and Applications (Springer)”, Vol. 60, Iss. 3, pp 689-726, October 2012.

[16] Harsh S., Haoyue L., Vijay K. D., Mansoor A., Prabir B., "Wireless Sensor Network Simulators A Survey and Comparisons", "International Journal Of Computer Networks (IJCN), Vol. 2, Iss. 5, pp. 249-265, 2011.

[17] Chhimwal P., Singh Rai D., Rawat D. “Comparison between Different Wireless Sensor Simulation Tools”, “Journal of Electronics and Communication Engineering (IOSR-JECE)”, Vol. 5, Iss. 2, pp.54-60, Apr. 2013.

[18] Patil A., Hadalgi P. M., "Evaluation of Discrete Event Wireless Sensor Network Simulators", "International Journal of Computer Science and Network (IJCSN)", Vol. 1, Iss. 5, pp. 1-10, Oct. 2012.

[19] The Manna Research Group. Mannasim Framework Classes Manual. [online], [cit. 25-10-2013]. Available from: www.mannasim.dcc.ufmg.br/download/mannasim-classes-manual.pdf/

[20] Jevtic M., Zogovic N., Dimic G., "Evaluation of Wireless Sensor Network Simulators”, "Proceedings of 17th Telecommunication forum TELFOR 2009”, Serbia, Belgrade, November 24 - 26, pp. 1303-1306.

[21] Christhu M.R., Chacko N., Major J., Shibin D., "A Comprehensive Overview on Different Network Simulators", "International Journal of Engineering and Technology (IJET)”, Vol. 5, No. 1, pp. 325-332, Feb-Mar 2013.

[22] Singh N., LalDua R., Mathur V., "Wireless Sensor Networks: Architecture, Protocols, Simulator Tool”, "International Journal of Advanced Research in Computer Science and Software Engineering”, Vol. 2, Iss. 5, pp. 229-233, May 2012.

[23] Musznicki B., Zwierzykowski P., "Survey of Simulators for Wireless Sensor Networks", "International Journal of Grid and Distributed Computing”, Vol. 5, No. 3, pp. 23-50, Sept. 2012.

[24] Korkalainen M., Sallinen M.. Karkkainen N., Tukeva P., "Survey of Wireless Sensor Networks Simulation Tools for Demanding Applications", "Fifth International Conference on Networking and Services, 2009 (ICNS '09)”, pp. 102-106, $20-25$ April 2009, Valencia.

[25] Abuarqoub A., Al-Fayez F., Alsboui T., Hammoudeh M., Nisbet A., "Simulation Issues in Wireless Sensor Networks: A Survey", "Proceedings of The Sixth International Conference on Sensor Technologies and Applications (SENSORCOMM 2012)", pp. 222-228, Aug. 19 - 24, 2012 - Rome, Italy.

[26] The Network Simulator ns-2: Documentation. Available from: http://www.isi.edu/nsnam/ns/ns-documentation.html.

[27] The Mannasim Research Group. Mannasim Framework Classes Manual. [online], [cit. 2010-10-19]. Available from: www.mannasim.dcc.ufmg.br/download/ mannasim-classes-manual.pdf/.

[28] Peng G., Tao L., Yanming C., Xingyao W., Yehia E., Edwards C., "The Measurement and Modeling of a P2P Streaming Video Service", Networks for Grid Applications- Lecture Notes of the Institute for Computer Sciences, Social Informatics and Telecommunications Engineering Volume 2, 2009, pp 24-34, ISSN: 1867-8211.

[29] Kurkowski S., Camp T., Colagrosso M., "MANET Simulation Studies: The Incredibles”, Mobile Computing and Communications Review, Vol. 1, No. 2, 2005. 